\title{
Entrevista a Andrés González, fundador y director del Consort Guitarrístico de Chile
}

\author{
Mauricio Gómez Gálvez y Andrés González
}

Conservatoire de musique et de danse d'Épinay-sur-Seine/GRMB Sorbonne | Francia Pontificia Universidad Católica de Valparaíso | Chile

\begin{abstract}
Resumen: Estructurada en tres partes, esta entrevista propone un recorrido por diversas temáticas en torno a una de las agrupaciones instrumentales más originales surgidas en las dos últimas décadas en América Latina: el Consort Guitarrístico de Chile (CGCL). Partiendo de la génésis de dicho consort, se abordan aspectos tales como la pedagogía de la guitarra, la identidad estética de la agrupación, la luthería empleada. La segunda parte trata acerca de las modalidades de difusión nacional e internacional del CGCL, el trabajo de grabación en estudio, además del estado del ámbito editorial chileno. Un diálogo sobre la continuidad de la labor guitarrística en el inestable contexto actual de crisis social, política y sanitaria, cierran la entrevista.
\end{abstract}

Palabras-clave: Andrés González, Consort Guitarrístico de Chile, Guitarra clásica, Música contemporánea, Música en la crisis social, política y sanitaria.

\begin{abstract}
Structured in three parts, this interview proposes a tour of various topics about one of the most original instrumental groups that emerged in the last two decades in Latin America: the Consort Guitarrístico de Chile (CGCL). Starting from the genesis of this consort, aspects such as the pedagogy of the guitar, the aesthetic identity of the group, the manufacture of instruments employed are addressed. The second part deals with the modalities of national and international dissemination of the CGCL, the work of recording in the studio, as well as the state of the Chilean editorial field. A dialogue on the continuity of guitar work in the unstable current context of social, political and health crisis, concludes the interview.
\end{abstract}

Keywords: Andrés González, Consort Guitarrístico de Chile, Classical Guitar, Contemporary Music, Music in the social, political and health crisis. 
S i hasta los años 1980 en Chile, la guitarra clásica acusaba un cierto retraso con respecto a países como Argentina, Uruguay o Brasil, un verdadero boom vendrá a reequilibrar dicha situación a partir de la década siguiente, insertando con fuerza la guitarra chilena dentro del ámbito internacional (OHLSEN, 1998, p. 5). Dentro de un panorama guitarrístico ya consolidado, con la presencia de numerosos solistas de talla internacional, vemos aparecer en las dos últimas décadas algunos proyectos innovadores dentro del medio musical chileno. Si el perfil solista aparece como mayoritario (“Una magnífica soledad está inscrita en los genes del guitarrista”, afirma Rafael Andia en un libro reciente [ANDIA, 2016, p. 96-97]), dicho modelo es contrabalanceado por propuestas alternativas que apuestan hacia la dimensión colectiva. Dentro de estas últimas, figura en primera línea el Consort Guitarrístico de Chile (denominado en lo sucesivo CGCL), fundado en la ciudad de Valparaíso por el guitarrista, compositor, filósofo y académico Andrés González (1977-), quien encarna de alguna manera la idea expresada por Frédéric Zigante, según la cual "un buen intérprete debe ser una combinación entre un atleta y un intelectual” (ZIGANTE, 2019, p. 18). La acción llevada a cabo por el CGCL en más de una década de existencia, así como su presencia tanto en su país de origen como en Europa, ameritan el presente diálogo. Este trabajo pretende llenar un vacío bibliográfico y contribuir a enriquecer las fuentes disponibles: en efecto, hasta el momento ningún diálogo con el entrevistado había sido publicado en revistas de musicología.

Desde un punto de vista metodológico, es necesario precisar que esta entrevista se sitúa a medio camino entre el cuestionario y la entrevista de investigación. Es decir que, tomando como punto de partida una serie de preguntas redactadas previamente, como en el primer procedimiento, se ha otorgado suficiente espacio al entrevistado para desarrollar abundantemente un discurso lineal, como en el segundo (BLANCHET et alii, 2000, p. 83). En fases posteriores de intercambio entre entrevistador y entrevistado ${ }^{1}$, fueron precisadas algunas ideas e incorporados algunos puntos complementarios, aunque sin alterar lo esencial de la producción inicial y sin perder de vista que "la 'subjetividad' del producto informativo generado es una propiedad de las entrevistas" (BLANCHET et alii, 2000, p. 82). En este sentido, siguiendo al dramaturgo David Lescot, entendemos la entrevista como un mecanismo de captación de un pensamiento, de un tono personal, dentro de un contexto determinado: "La entrevista permite alcanzar conjuntamente lo íntimo y lo histórico, de alzar lo

\footnotetext{
${ }^{1}$ Ambos figuran como coautores, en conformidad con la línea editorial de la Revista Vórtex.
} 
íntimo a la altura de la historia y, de retorno, encarnar lo histórico en figuras humanas. La entrevista permite además aproximarse al estilo de los que hablan” (LESCOT, 2015, p. 18).

En la siguiente entrevista, realizada a distancia -entre París y Valparaíso- y, como ya lo hemos precisado, por fases sucesivas entre junio y julio de 2020 (y revisada en el mes de octubre), se abordan temas que atañen no solamente a la guitarra y al medio guitarrístico, sino que conciernen también al ámbito, más amplio, de la música contemporánea y el quehacer musical en general en Chile y Latinoamérica.

\section{Génesis, conformación y desarrollo de una formación instrumental original}

Pregunta: Al momento de concebir el proyecto del Consort Guitarrístico de Chile, tú ya tenías un amplio recorrido como guitarrista solista, en un dúo de guitarra y charango y junto a diversos ensambles ${ }^{2}$, además de un considerable trabajo de compositor ${ }^{3}$. ¿Cómo surge la idea de creación de un consort de guitarras, en qué contexto preciso y con qué objetivos?

A.G.: Trabajábamos con un colega guitarrista en una escuela de música en San Felipe ${ }^{4}$, donde teníamos muchos estudiantes de guitarra. Habíamos iniciado un proceso de ir sistematizando y apoyando los ensambles de la escuela, había interés de parte de la dirección de crear una orquesta (en pleno apogeo de las orquestas juveniles-infantiles) y formamos una orquesta de guitarras. Se nos ocurrió mandar a diseñar y construir guitarras de diverso registro para poder transcribir obras orquestales directamente sin tener que hacer octavaciones, tanto en el registro agudo como grave. Así mandamos a hacer, gracias a un proyecto FNEA 5 , 4 tipos de guitarras, 4 sopranos, 2 altos, 2 bajos y 2 contrabajos, a las que se añadieron las guitarras estándar y armamos una orquesta de unos 15 niños. La orquesta funcionó muy bien y en poco tiempo hicimos varios conciertos. A partir de esa experiencia ideamos armar un Consort profesional.

\footnotetext{
${ }^{2}$ En aquel período, Andrés González ya había formado parte de agrupaciones tales como el grupo de fusión y experimentación latinoamericana Transiente (como multiinstrumentista y compositor) o el Dúo Noesis. Para este último, destaca su transcripción de las Sechs kleine Klavierstücke, op. 19 de Arnold Schönberg, para charango y guitarra.

${ }^{3}$ Acerca de la trayectoria de González, cf. GÓMEZ GÁLVEZ, 2017 y 2020.

${ }^{4}$ Ciudad de la Región de Valparaíso, Chile.

${ }^{5}$ Se trata del antiguo Fondo Nacional de Escuelas Artísticas. Actualmente, dicho fondo concursable del Gobierno de Chile ha sido reabsorbido y transformado en el Fondo de Fomento al Arte en la Educación (FAE).
} 
Antecedentes de este tipo de formación no son muchos. Dentro de los que nosotros tomamos como referencia, está un quinteto femenino alemán llamado Guitarrisima, pero ellas usan una guitarra soprano (Oktavgitarre), una contrabajo (Kontrabass-Gitarre) y tres guitarras estándar. También conocimos el trabajo que realiza la Niibori Orchestra en Japón, que reúne a cerca de 100 guitarristas y varios ensambles. En Latinoamérica teníamos conocimiento de la Orquesta de Guitarras Sonantas Habaneras de Cuba, bajo la dirección de Jesús Ortega, quienes además de las guitarras estándar incorporan requintos y guitarras bajas. El 2013 aparece la Orquesta de Guitarras de Chile, quienes básicamente usaban guitarras estándar más contrabajo y entiendo que posteriormente incorporan otros instrumentos de diverso registro.

Pregunta: Lo que me parece particularmente original es la actualización que han realizado de la antigua idea del consort o, más precisamente de un whole consort, estableciendo así un interesante vínculo histórico con una práctica instrumental pasada, pero a partir de un instrumento moderno.

A.G.: Sí, esta idea de ampliar la guitarra hacia toda su familia es algo que se había perdido para las cuerdas pulsadas con el correr de los siglos (en el Renacimiento había varios tipos de laúd y tiorbas con distintas afinaciones, pero al parecer su uso como consort no fue muy regular). Entonces la idea la sacamos más bien de los antiguos consort de violas da gamba, traduciendo esa formación a la guitarra moderna y manteniendo la lógica de construcción de otros instrumentos de familias como los saxofones (estandarizados en dos afinaciones y con un diseño de construcción pensado para que cualquier instrumentista pueda, luego de un corto tiempo de adaptación, ejecutar cualquiera de las guitarras) y así facilitar la transposición y lectura de partituras. En nuestro caso, la afinación que determinamos para las guitarras consiste en que tres de ellas mantienen la afinación tradicional mi,

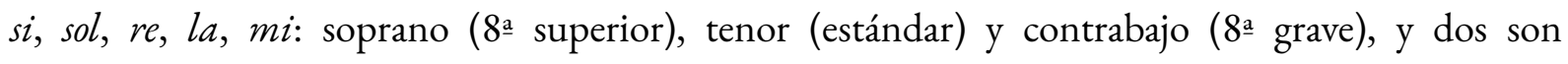
transpositoras, la alto y bajo, cuya afinación es $s i$, fa\#, re, la, mi, si, a distancia de una octava entre ellas. Gracias a esta configuración el consort puede cubrir todo el registro sonoro, sin necesidad de realizar octavaciones o transposiciones en el caso de la transcripción de obras originales. Pero quizás lo más interesante sea el hecho de que, además del registro, cada guitarra tiene un timbre característico que colorea la sonoridad general del Consort.

Pregunta: Si la configuración instrumental del consort se ha mantenido estable, diversos miembros han integrado sucesivamente la agrupación. ¿Podrías hablarnos de sus integrantes y la 
evolución del consort en este sentido? ¿Cómo han logrado conservar una identidad de grupo y una dirección estética en más de una década de existencia, a pesar de la rotación de sus miembros?

A.G.: En la formación original estaban Rodrigo Erazo, Moa Edmunds, Sebastián Avello, Ramón Moncada y yo. Desde entonces han pasado varios guitarristas, como Pablo Palacios, Ignacio Barra, Marco Carmona y Darío Vargas, hasta la actual formación con, además de Erazo y yo, Claudia Morán, Rodrigo Moyano y Oscar Ovalle. Creo que la mantención del sello y características del Consort se deben, por un lado, a que definimos bien desde el comienzo tres líneas de trabajo, que se han mantenido pese a los múltiples cambios de integrantes hasta ahora, las cuales son la transcripción de música europea, principalmente orquestal, la música latinoamericana y obras originales, sobre todo de compositores chilenos y, por otro lado, a que dos de los fundadores del Consort nos hemos mantenido estables y hemos garantizado que el ensamble esté constantemente activo. Incluso durante mi estadía en el extranjero, entre el 2011 y 2014, el Consort continuó su actividad. Por otro lado, la música de cámara ha sido para nosotros sinónimo de "camaradería”, lo cual por supuesto repercute positivamente en la permanencia del proyecto por el establecimiento de lazos de amistad.

Pregunta: A propósito de esta forma de trabajo en conjunto, ¿en tu opinión, existen características distintivas de la enseñanza y del medio guitarrístico en Valparaíso -del cual ustedes forman parte- con respecto a lo que se desarrolla en Santiago?

A.G.: Yo diría que más que diferencias en las metodologías de enseñanza, que de hecho las hay, me da la impresión que hay una relación distinta entre los colegas y eso termina repercutiendo en la enseñanza de la guitarra. Creo que en general los guitarristas porteños ${ }^{6}$ somos más gregarios que los santiaguinos y estamos menos preocupados de la competencia, por eso hay varias agrupaciones de guitarristas en la Quinta Región ${ }^{7}$ que en vez de competir colaboran entre sí individual y colectivamente. Esto repercute en las aspiraciones de los guitarristas y consecuentemente en las metodologías de enseñanza-aprendizaje. Cuando la preocupación y la finalidad es la música y su disfrute, y no tanto la velocidad de las semicorcheas o la perfección técnica de la ejecución, creo que hay mucha mayor conexión entre los músicos y nos sentimos más al servicio de las obras que al revés. Lo cual no quiere decir que los colegas porteños no tengan un perfil solista y que no ganen concursos

\footnotetext{
${ }^{6}$ « Porteño » es la denominación dada al habitante de Valparaíso, importante ciudad portuaria.

${ }^{7}$ Región de Valparaíso.
} 
de vez en cuando, sólo que la camaradería es algo que cultivamos constantemente y eso favorece mucho el desarrollo de la música de cámara por sobre el rol solista. No quiero decir con esto que en Santiago sean individualistas, pero allá se observa mucho más que en nuestro medio ese perfil de solista ganador, centrado en la interpretación de música tradicional.

Pregunta: Como ya lo hemos evocado más arriba, un aspecto fundamental del CGCH es su instrumental, constituido por guitarras de diferentes registros. Quisiera que te refirieras ahora al ámbito específico de la luthería. ¿Con qué guitarras tocan? ¿Cómo se operó la elección de un luthier en particular para su confección?

A.G.: Las guitarras son, como te decía antes: guitarra soprano, afinada una octava más aguda que la estándar, guitarra alto, afinada una quinta por encima de la estándar, guitarra tenor (estándar), guitarra bajo, una cuarta por debajo de la estándar y guitarra contrabajo, una octava más grave que la estándar. Cuando hicimos el proyecto original, le consultamos a varios luthiers chilenos y finalmente fue Juan Carlos Moraga ${ }^{8}$ el que terminó diseñando y construyendo las guitarras. Nosotros no contábamos en ese momento con ningún instrumento como para copiar los tamaños y proporciones, solamente fotos y videos de otras guitarras en Oriente y Europa. Entonces el luthier prácticamente tuvo que diseñarlas desde cero. Lo que más nos importaba era que fuesen instrumentos lo más parecidos a una guitarra clásica, con tal de que cualquier guitarrista pudiese tocarlas sin mayores dificultades. Entonces el diapasón tiene un ancho parecido a la estándar (sólo un par de milímetros de diferencia en virtud del diámetro de las cuerdas) y las cajas de resonancia guardan también relación con el tamaño estándar, así no dificultan demasiado la interpretación. También quisimos facilitar la lectura musical con estos instrumentos y, como mencioné antes, tanto la alto como la bajo son transpositoras, todas leen en llave de sol como si fuesen guitarras estándar.

Una de las problemáticas de un proyecto de estas características, y que es una de las preguntas recurrentes que se nos suele hacer, es acerca de la obtención de cuerdas específicas para las afinaciones, tamaños y tensiones de las guitarras nuevas. Para esto se deben considerar varios factores adicionales como el diámetro de la cuerda, su largo vibrante y el material. Por ejemplo para la primera cuerda de guitarra soprano no sirven las cuerdas de charango porque tienen un diámetro inferior a lo que se requiere para una tensión adecuada, pese a tener la misma afinación y largo similar. Para la contrabajo

\footnotetext{
${ }^{8}$ Luthier radicado en la ciudad de Valdivia, en el sur de Chile.
} 
no sirven las cuerdas de bajo acústico porque se requiere nylon y normalmente las de bajo son metálicas. Por fortuna hoy en día es posible encontrar en diversas latitudes la cuerda apropiada para cualquier instrumento; la mayoría hemos tenido que encargarlas a Alemania, pero actualmente podemos conseguir algunas con empresas locales que las importan.

Pregunta: Dentro de las primeras obras interpretadas por el CGCH se encontraba la versión inicial de Aión, primera composición de tu autoría concebida especialmente para la agrupación, y a partir de la cual comienzan a estructurar su repertorio. ¿Con qué criterios han ido conformando el repertorio del Consort?

A.G.: Como mencionaba más arriba, una de las líneas de trabajo iniciales, junto con la transcripción y la música latinoamericana, y que se ha mantenido hasta ahora, es el estreno de obras originales para la agrupación. Aión no fue la primera obra escrita para el Consort, pero sí la primera en ser estrenada. Su primera versión contaba con electrónica en tiempo real y la versión definitiva incluye ensamble 9 . En esta línea, en que hemos ejecutado unas 10 obras, nos ha interesado trabajar lo más estrechamente posible con los compositores, entre ellos contamos a Felipe Pinto d'Aguiar, Gabriel Matthey, Pedro Álvarez, y en estos momentos está escribiendo para nosotros el compositor andino Francisco Silva. En general nuestro interés es abordar diversas estéticas y estamos abiertos a recibir obras que incorporen técnicas contemporáneas de ejecución y electrónica. En las otras líneas de trabajo vamos escogiendo las obras de manera bien intuitiva y siguiendo el gusto personal de los integrantes, y en algunos casos incluso transcribiendo del audio cuando no existe partitura de por medio, como en el caso de la pieza Mitimaes de Claudio Araya. En el caso de obras de la tradición occidental, buscamos piezas que permitan una transcripción directa y aquellas en las que podamos respetar al máximo la partitura original. Intentamos respetar el espíritu de las piezas y en el caso de obras orquestales, que presentan múltiples líneas instrumentales, tratamos de no perder música de la partitura original, por supuesto resguardando los límites de ejecución, como por ejemplo, el Concierto Brandenburgués $\mathrm{n}^{\circ} 4 \mathrm{de}$ J. S. Bach.

\footnotetext{
${ }^{9}$ La primera versión de Aión data de 2008, la segunda de 2013. Acerca de esta obra, cf. GÓMEZ GÁLVEZ, 2016, p. 202203.
} 


\section{Dif usión y circulación internacional}

Pregunta: La labor de difusión artística del consort la han llevado a cabo de diversas formas y medios. Comencemos por referirnos a la modalidad de más antigua data, históricamente hablando: los conciertos. ¿Qué importancia asignas a esta forma de difusión tradicional y qué lugar ha tenido en la actividad del CGCH?

A.G.: Creo que el concierto tradicional es importante para nosotros, pero valoramos más el proceso de ensayos e incluso el de juntarse a hablar de música. Yo diría que el CGCL no ha tenido una presencia demasiado constante en escenarios. Me parece que en general nuestra aparición es más bien esporádica. Esto, lejos de ser una merma, creo que genera un equilibrio entre el trabajo de música de cámara, que es bien minucioso y tiende más a la calidad que a la cantidad, la educación musical y una presencia en la escena local. Consecuentemente, las ocasiones para mostrar nuevas piezas se ven disminuidas, es decir, tratamos de no saturar de presentaciones mostrando el mismo repertorio, sino que trabajamos más bien con proyectos de programa que vamos presentando y adecuando si es necesario.

Pregunta: Otro medio de difusión esencial de vuestro trabajo han sido las grabaciones. Luego de un primer CD de características bastante eclécticas ${ }^{10}$, el segundo disco del CGCH -titulado Micorriza $(2016)^{11}$ - marca un giro en la discografía, tornándose netamente hacia la creación de nueva música chilena, con obras concebidas especialmente para ustedes. Ese disco contiene además una nueva versión de Aión, grabada junto al ensamble f(r)actura, otro de tus proyectos paralelos ${ }^{12}$. Respecto a la música registrada en disco, Glenn Gould afirmó alguna vez lo siguiente: "Yo no considero que un disco deba ser la representación sobre una tarjeta postal de un concierto, ni la momificación de una interpretación o un instante particular” (GOULD, 1983, p. 114). Considerando que el repertorio que abordan en el segundo disco es de gran complejidad, ¿cómo proceden ustedes al momento de grabar: a la manera de Gould, utilizando todas las posibilidades de montaje que ofrece el estudio moderno, o más bien por selección de tomas de largo aliento? Me

\footnotetext{
${ }^{10}$ El primer disco del CGCL contiene obras de Frédéric Chopin, Edvard Grieg, Maurice Ravel, además de los chilenos Patricio Wang, Claudio Araya y Andrés González.

${ }^{11}$ Para más detalles acerca de este CD, cf. GÓMEZ GÁLVEZ, 2016.

${ }^{12}$ Cf. GÓMEZ GÁLVEZ, 2019 y 2016.
} 
interesaría conocer tu posicionamiento frente a la música grabada y saber cómo evalúas la labor realizada por el CGCH en este ámbito.

A.G.: Creo que la grabación te pone en otro nivel de escucha, diferente al del concierto. No creo que uno sea mejor que otro, simplemente ambos ponen los acentos en diferentes aspectos y desde el punto de vista de la interpretación uno puede aprovechar esas diferencias para remarcar aspectos, incluso técnicos, diversos. La grabación permite mayores grados de precisión, por la posibilidad de edición, lo que es comprensible, ya que es un soporte que está hecho para ser escuchado múltiples veces y uno puede trabajar aspectos sonoros imposibles de manejar en vivo. El concierto es menos preciso pero tiene una componente emocional y de vínculo con los músicos y el público, que no se tiene en el estudio, y la “perfección” se da al interior de esa relación. El Consort tuvo dos experiencias diferentes en ambas producciones: primero, por el repertorio, y segundo, por la manera en que fueron grabados. El primer disco fue una absoluta autoproducción, grabado íntegramente en mi estudio casero y en un momento en que nos era imposible grabar todos juntos, con lo que tuvimos que registrar cada guitarra por separado y luego montar. En el segundo, trabajamos con un ingeniero en sonido e intentamos hacer todo más camarístico pero también hubo edición. Estamos trabajando en un tercer CD más en la línea ecléctica del primero, pero esta vez grabando todos juntos. En este medio de difusión, creo que se ha mantenido lo que te decía a propósito del concierto, en el sentido de preferir trabajos acotados pero atendiendo a los detalles.

Respecto a la segunda parte de tu pregunta, aún entendiendo que el todo no es la simple suma de todas las partes, debo decir que las grabaciones que hemos hecho últimamente, no sólo con el Consort sino también con $\mathrm{f}(\mathrm{r})$ actura, tienen de todo, desde el intento de grabar grandes secciones para mantener los arcos, hasta la edición detallada de partes más pequeñas. Entonces en ese sentido, sí utilizamos todas las herramientas que ofrece el estudio moderno, como Gould, sobre todo cuando tenemos la posibilidad de trabajar con nuestras propias herramientas y no dependemos de horarios fijos ni límites en presupuesto. Pero por supuesto, en el ideario de todos está el anhelo de hacer tomas largas que queden bien en un solo aliento, y eso a veces se cumple.

Pregunta: En alguno de nuestros intercambios $\operatorname{pasados}^{13}$ ya tuvimos la oportunidad de evocar el tema de la "interignorancia” musical persistente entre países latinoamericanos, y en la situación

\footnotetext{
${ }^{13}$ Cuestionario de septiembre 2017 entre los coautores de esta entrevista.
} 
paradojal de conocernos mutuamente en tanto músicos a través de esta suerte de centros neurálgicos y correas de transmisión que siguen constituyendo en buena parte Europa y EEUU hasta el día de hoy. En este sentido, ¿qué vínculos han podido establecer con otros medios musicales del continente? ¿Han tenido contactos con Brasil, por ejemplo, país de gran tradición guitarrística?

A.G.: Lamentablemente en nuestro caso, como en muchos otros, ha imperado esta lógica de interignorancia que habíamos abordado antes y nuestros contactos han sido más bien con el viejo continente que con el resto de América; no obstante, hemos tenido contacto con algunos importantes guitarristas latinoamericanos como Jesús Ortega, de Cuba, pero no hemos profundizado demasiado esos lazos. Parece ser que nos han absorbido otras preocupaciones en diversos ámbitos, como la educación, la composición y proyectos paralelos como el Estudio Modular de Música Actual $(E M M A)^{14}$. Me parece que, y esto a modo de autocrítica, es una tarea pendiente extrapolar el sentido de camaradería que cultivamos internamente, a otras esferas y establecer lazos con otros músicos y agrupaciones del continente.

Pregunta: La música contemporánea chilena, salvo excepciones y a pesar de diversas iniciativas, ha sufrido de un déficit histórico de difusión en el plano de la edición de partituras. Las obras para guitarra no son una excepción al respecto. Aunque internet ha remediado en gran parte este problema, ¿consideras necesario el desarrollo del mundo editorial en el país? ¿Existe la posibilidad de crear una rama editorial a través de la articulación entre el CGCH y el EMMA?

A.G.: Efectivamente, creo que ha habido en los últimos años un cambio de foco en la difusión de partituras en general, pasando del papel al PDF, pero creo que esa tendencia está empezando a revertirse y lo que se observa es una división entre las grandes editoriales tradicionales y un montón de editoriales pequeñas que están surgiendo, varias de ellas con interés en la música actual. En Valparaíso existe la Editorial Cadenza, con la que hemos hecho algunos trabajos con el ensamble $\mathrm{f}(\mathrm{r})$ actura, aún no en el plano de la publicación, pero sí con proyectos específicos con algunos ejemplares de muestra, como el proyecto Freund Stein // El Amigo Piedra que hicimos durante el 2018. Me parece que es muy importante apoyar el desarrollo del mundo editorial en Chile, sobre todo de música nueva, porque creo que es más que un simple soporte (como puede ser el digital, que es

\footnotetext{
${ }^{14}$ El Estudio Modular de Música Actual (EMMA), fue fundado por Andrés González el año 2014, con el fin de promover la nueva creación musical local. Esta institución independiente sostiene diversas acciones, intrínsecamente asociadas, tales como el ensamble f(r)actura y el Sello Modular.
} 
únicamente un soporte), es más que una réplica, es la posibilidad de que el ejemplar se vuelva único, y eso sólo lo da el contacto con el objeto físico. Creo que es lo mismo que pasa con el CD que se resiste a morir, a la vez que reaparecen el vinilo y el cassette, no por simple fetiche, sino por ese valor que se superpone al contenido y que se transforma en contenido (no simple continente). Con $\mathrm{f}(\mathrm{r})$ actura y el CGCL hemos tenido muchas intenciones de publicar libros de partituras, pero por ahora son un anhelo, ya que requieren de un gran esfuerzo de producción. Tal vez pronto...

\section{La guitarra en tiempos de crisis}

Pregunta: Durante la grave crisis política que se inicia en Chile en octubre de 2019 con el denominado "estallido social", las manifestaciones estuvieron marcadas por la presencia de la guitarra como símbolo de lucha, a menudo asociada a la figura de Víctor Jara. Ello me recuerda que en el repertorio del CGCH figuran justamente tus Disvariaciones sobre un tema de Victor Jara. ¿Participó el CGCH en las actividades musicales ligadas a dicho movimiento social?

A.G.: Todos tuvimos alguna participación activa en eventos culturales asociados a la revuelta. Con algunos de los miembros del Consort fuimos parte de una gran orquesta que se armó entre músicos de Valparaíso, Viña, Quilpué y Villa Alemana, interpretando parte del cancionero tradicional de protesta, con música de Víctor Jara, Violeta Parra y, por supuesto, El Pueblo Unido de Sergio Ortega. Yo personalmente retomé el saxofón para tocar en la calle en diversos conciertos, algunos más o menos improvisados. Con el CGCL tocamos en el Festival Relincha ${ }^{15}$, el 22 de noviembre, en donde presentamos una versión de la Pieza IV para ensamble y electrónica que compusimos en conjunto con Enrique Schadenberg ${ }^{16}$, la que unimos a Litoral de Felipe Pinto d'Aguiar y finalizamos con El Derecho de Vivir en Paz de Víctor Jara, en cajita musical.

A este respecto puedo añadir que el rol que ha jugado el arte y, en particular, la música en todo este complejo proceso, ha sido el de una especie de "catalizador" de pensamientos, percepciones y emociones que habían estado latentes o en muchos casos invisibilizados durante años. Por otro lado, muchos artistas habíamos caído en la lógica perversa de la competencia neoliberal, sobre todo en lo

\footnotetext{
${ }^{15}$ Festival de música experimental organizado anualmente en la ciudad de Valdivia.

${ }^{16}$ Performance disponible en https://www.youtube.com/watch?v=oPqggzX7dbo (consultado el 23/07/2020).
} 
que respecta a los mecanismos de financiamiento del arte y la cultura en Chile, asunto que es urgente revisar y cambiar. Creo que como sector cultural tenemos una oportunidad de participar activamente de las decisiones del país y, consecuentemente, del poder, y esto pasa necesariamente por la conformación de colectivos e instituciones capaces de canalizar la producción artística y distribuir los recursos de forma más equitativa.

Pregunta: Actualmente vivimos tiempos de particular dificultad con la crisis sanitaria ligada a la pandemia de coronavirus, la que golpea fuertemente el medio artístico, con conciertos y festivales anulados, con restricciones de circulación nacional e internacional, con proyectos de grabación abortados, etc. ¿Cómo enfrenta una agrupación como el CGCH estos tiempos de gran fragilidad?

A.G.: Por fortuna no vivimos del concierto. De hecho, como te comentaba, el proyecto siempre se definió como "artístico", en donde la única pretensión fue siempre desarrollar y contribuir a la difusión de música de arte para este formato. Entonces los conciertos son solamente la etapa final de un relativamente largo proceso de montaje y af iatamiento de un programa. Generalmente, los pagos que recibimos no son más que un apoyo para seguir trabajando, y alcanza para comprar cuerdas, efectuar reparaciones y cosas por el estilo. Por supuesto que echamos de menos tocar y por sobre todo juntarnos a ensayar y conversar, que es algo que por suerte siempre hemos mantenido. Por sobre todo somos un grupo de amigos y eso se refleja en la música. En este tiempo solamente logramos hacer uno de esos típicos videos en que aparece cada uno en su casa ${ }^{17}$. Hemos intentado hacer ensayos por internet, pero es demasiado problemático... Ahora, nosotros tenemos la suerte de trabajar en instituciones de enseñanza musical y si bien en general los ingresos han disminuido por no tener la posibilidad de hacer conciertos en vivo, no nos hemos sentido tan fuertemente afectados económicamente, no así muchos colegas cuya fuente principal de ingresos son las presentaciones en vivo. Esto nos devuelve a la urgente revisión de la lógica del financiamiento cultural.

Pregunta: El CGCH aparece como un islote creativo y audaz en un medio guitarrístico volcado fuertemente hacia la competitividad agresiva de los concursos internacionales y su indisociable búsqueda de un virtuosismo asociado esencialmente a estéticas pasadas ${ }^{18}$. ¿Cómo ves el porvenir de la guitarra en Chile?

\footnotetext{
${ }^{17}$ Se trata de una versión de Lo que vendrá de Astor Piazzolla. Disponible en: https://www.youtube.com/watch?v=RRS9ikQxikQ (consultado el 24/07/2020).

${ }^{18}$ Acerca de los concursos de guitarra en Chile y el extranjero, cf. Ohlsen, 1998.
} 
A.G.: Concuerdo con que existe una tendencia mayoritaria en la vía de un estereotipo de guitarrista virtuoso, ganador de concursos y centrado en un repertorio tradicional, a la que hay que reconocer en todo caso un desarrollo técnico efectivo aunque en extremo acotado, y que esta tendencia lamentablemente se instaura en las academias en las que se formatea a los nuevos músicos. Sin embargo, también veo surgir otras tendencias, como la llamada "Nueva Guitarra Chilena", con músicos como "Chicoria” Sánchez" o Javier Contreras, que por supuesto están lejos de lo que hacemos con el Consort, pero son una alternativa diferente al guitarrista de concurso. A mí me parece que lo que urge en estos momentos en la guitarra, no sólo en Chile, sino también a nivel mundial es hacerla entrar en el siglo XXI. Sabemos que la guitarra históricamente ha ido retrasada cerca de un siglo, viviendo su romanticismo en pleno siglo XX y ha sido en general reticente a los nuevos lenguajes musicales, sin embargo creo que puede "saltarse" el siglo XX y entrar de lleno al mundo del sonido, la experimentación y reflexión en torno al sonido, y no sólo abordar las "formas musicales” y sus transformaciones. Es hora de que la guitarra aborde en serio el llamado que hace algunos años me indicaba Gabriel Matthey, en el sentido de que la guitarra debe ser el instrumento del siglo XXI, así como el piano fue el instrumento del siglo XIX. Estoy convencido de que el futuro de la guitarra irá de la mano de desarrollos diversos, tanto desde la composición y el florecimiento de nuevas técnicas instrumentales, como de la luthería y la incorporación de instrumentos tradicionales latinoamericanos, como el charango, el cuatro, el tiple y muchos otros.

Pregunta: Para cerrar esta entrevista, y en espera de tiempos mejores, ¿podrías hablarnos brevemente de los proyectos futuros del CGCH?

A.G.: Terminamos una grabación poco tiempo antes de la pandemia, que espero podamos publicar prontamente, que contiene obras de Piazzolla, Brahms, Bach, Gismonti y Dowland. Queremos hacer un segundo programa de compositores chilenos, por supuesto también con grabación. Tenemos una obra de Gabriel Matthey y dos obras en curso de José Miguel Arellano y Francisco Silva, a las que queremos sumar un par de piezas más para completar el ciclo. También hemos tenido últimamente un acercamiento a la improvisación, lo cual es una práctica que sólo habíamos experimentado en forma muy acotada en mi obra Aión, y que nos gustaría desarrollar de mejor manera una vez que podamos recuperar nuestras actividades.

\footnotetext{
${ }^{19}$ Juan Antonio Sánchez (1965-), compositor y guitarrista chileno.
} 


\section{REFERENCIAS}

ANDIA, Rafael. Labyrinthes d'un guitariste. París: L'Harmattan, 2016.

BLANCHET, Alain, GHIGLIONE, Rodolphe, MASSONAT, Jean, TROGNON, Alain, Les techniques d'enquête en sciences sociales. Observer, interviewer, questionner. París: Dunod, 2000.

CONSORT GUITARRÍSTICO DE CHILE. Micorriza. Valparaíso: EMMA/Fondo para el fomento de la música nacional, 2016. CD.

ENSAMBLE F(R)ACTURA. $f(r)$. Valparaíso: Sello Modular, 2019. CD.

GÓMEZ GÁlVEZ, Mauricio. CD Consort Guitarrístico de Chile, Micorriza, Valparaíso, EMMA/Fondo para el fomento de la música nacional, 2016 [reseña]. Resonancias. Revista de investigación musical, Santiago, v. 20, n. 39, pp. 201-203, julio-noviembre 2016.

GÓMEZ GÁLVEZ, Mauricio. Les formes d'appropriation dans la musique savante chilienne, $X X^{e}$ $X X I^{e}$ siècles: transfert culturel, acculturation, métissage. 2017. Tesis de Doctorado en musicología. Université Paris-Sorbonne, París.

GÓMEZ GÁLVEZ, Mauricio. Ensamble f(r)actura. 2019.f(r). Valparaíso: Sello Modular. CD [reseña]. Resonancias. Revista de investigación musical, Santiago, v. 23, n. 45, pp. 270-273, julionoviembre 2019.

GÓMEZ GÁLVEZ, Mauricio. La musique chilienne dans la mondialisation. Hermès. La Revue, París, n. 86, pp. 237-243, 2020.

GOULD, Glenn. Le dernier puritain. Écrits (tome I) réunis, présentés et traduits de l'anglais par Bruno Monsaingeon. París: Fayard, 1983.

LESCOT, David. Ceux qui restent. Entretiens avec Wlodka Blit-Robertson et Paul Felenbok. París: Gallimard, 2015.

OHLSEN, Óscar. En torno a los concursos de guitarra. Conversaciones con Ernesto Quezada y Luis Orlandini. Resonancias. Revista de investigación musical, Santiago, v. 2, n. 2, pp. 5-16, mayo 1998.

ZIGANTE, Frédéric. Frédéric Zigante: "Un bon interprète doit être la combinaison entre un athlète et un intellectuel”. Guitare Classique, n. 88, pp. 16-18, septiembre-noviembre 2019. Entrevista efectuada por Orestis Kalampalikis. 


\section{SOBRE LOS AUTORES}

Mauricio Gómez Gálvez es Doctor en musicología por la Université Paris-Sorbonne (2017), además de guitarrista y pedagogo. Ha publicado en las revistas Transposition. Musique et sciences sociales; Anuario Musical; Resonancias. Revista de investigación musical; Amérique Latine Histoire et Mémoire. Les Cabiers ALHIM; Hermès; y aportado en diversos libros colectivos. Desde 2019 integra la Red Trayectorias: Música entre América Latina y Europa, dedicada al estudio de la circulación musical transnacional. En Francia, participa en las actividades del GRMB (IReMus/Sorbonne) y otros grupos de investigación. Paralelamente a sus actividades de investigador, se desempeña actualmente como docente en el Conservatoire de musique d'Épinay-sur-Seine. ORCID: https://orcid.org/0000-0002-8586-9332. E-mail: mauricio gomezgalvez@yahoo.fr

Andrés González es Máster en composición por la Hochschule für Musik Stuttgart. En la PUCV, obtiene un Magíster en filosofía, el título de Intérprete superior en guitarra y una Licenciatura en Ciencias y Artes Musicales. Como intérprete, ha realizado conciertos en Chile y Europa. Sus composiciones se han estrenado en Chile, Argentina, Alemania, España y Francia, y han sido publicadas bajo el sello SVR, el Netlabel Pueblo Nuevo y Sello Modular. Desde 2015 es docente en la Pontificia Universidad Católica de Valparaíso. Es fundador y director del Estudio Modular de Música Actual (EMMA)

y director artístico del ensamble f(r)actura. ORCID: https://orcid.org/0000-0003-4385-1035._E-mail: andonzalez77@gmail.com 